\title{
Population Structure of Fusarium graminearum Isolated from Different Sources in One Area over the Course of Three Years
}

\author{
Donatas Sneideris, ${ }^{1, \dagger}$ Algirdas Ivanauskas, ${ }^{1}$ Petras Prakas, ${ }^{1}$ Dalius Butkauskas, ${ }^{1}$ Olga Treikale, ${ }^{2}$ Grazina Kadziene, ${ }^{3}$ \\ Neringa Rasiukeviciute, ${ }^{3}$ Jurgita Kelpsiene, ${ }^{3}$ and Skaidre Suproniene ${ }^{3}$ \\ ${ }^{1}$ Nature Research Centre, Akademijos str. 2, LT-08412, Vilnius, Lithuania \\ ${ }^{2}$ Latvian Plant Protection Research Centre, Struktoru 14a, LV-1039, Riga, Latvia \\ ${ }^{3}$ Institute of Agriculture, Lithuanian Research Centre for Agriculture and Forestry, Instituto al. 1, Akademija, LT-58344, Kedainiai \\ distr., Lithuania \\ Accepted for publication 25 March 2020.
}

\begin{abstract}
Fusarium head blight (FHB) is an important crop disease worldwide and is mainly caused by members of the Fusarium graminearum species complex. F. graminearum sensu stricto is the most common cosmopolitan and predominant FHB causal agent in Europe. Thus far, the majority of studies have focused on the primary hosts (wheat and barley) of this pathogen, while the relationships between other sources of infection remain unclear. We monitored and sampled test fields over the course of 3 years and acquired $804 \mathrm{~F}$. graminearum isolates from different sources: primary hosts and other plant species included in the crop rotations, weeds from the test fields, decaying plant residue, soil samples, and crop seed. Of these isolates, $73.3 \%$ had the 15 -acetyldeoxynivalenol genotype
\end{abstract}

ABSTRACT

Fusarium head blight (FHB) is a devastating disease of cereals on a global scale. In addition to severe yield losses, it often results in dangerous mycotoxin accumulation in harvested grain. Although FHB can be caused by several species in the genus Fusarium, the biggest and most common contributor to this disease are members of the Fusarium graminearum species complex (FGSC) (Aoki et al. 2012; Schothorst and van Egmond 2004; Yli-Mattila et al. 2008, 2009). The FGSC consists of 16 closely related species. However, its flagship species, $F$. graminearum sensu stricto, is the most widespread and cosmopolitan as well as predominant and the main cause of FHB in Europe (Boutigny et al. 2014; Somma et al. 2014; Wang et al. 2011).

Members of the FGSC usually produce one of three different types of B trichothecenes: nivalenol (NIV), 15-acetyldeoxynivalenol (15$\mathrm{ADON})$, or 3-acetyldeoxynivalenol (3-ADON). All three are able to inhibit the eukaryotic protein production system and are dangerous to humans and animal livestock. The emergence rates and dominance of any given chemotype in FGSC populations varies in different regions. It has been shown that populations expressing different types of toxin also differ in their physical properties. Populations exhibit different growth rates, aggressiveness toward host plants, and resistance to fungicides (Aamot et al. 2015; Aoki et al. 2012; Wang et al. 2011). Thus, tracking these chemotypes is important in order to better understand the properties of this pathogen and to aid the search for means to prevent and control the disease.

${ }^{\dagger}$ Corresponding author: D. Sneideris; donatas.sneideris@gmail.com

Funding: This study was funded by the Lithuanian Research Council through the National Research Program "Sustainability of agro, forest and water ecosystems", grant number SIT-05/2015.

The author(s) declare no conflict of interest.

(c) 2020 The American Phytopathological Society and $26.7 \%$ had the 3 -acetyldeoxynivalenol genotype. F. graminearum isolation rates from weeds $(>50 \%)$ were much higher than from soil $(<10 \%)$ or decaying plant matter $(4 \%)$. Variable number of tandem repeat markers were used for population analysis. Noticeable genetic differentiation was detected between isolates from living plants and soil biome. In contrast, absence of any noticeable division between primary and alternative plant host communities indicates the importance of weeds and other segetal plants for FHB control and prevention.

Keywords: Fusarium graminearum, Fusarium head blight, mycology, population biology, population genetic variation, VNTR

Over the last decade, there have been increasing numbers of reports that $F$. graminearum has spread to northern parts of Europe, where it replaces other, previously dominant species such as F. culmorum or F. avenaeceum (Aamot et al. 2015; Parikka et al. 2012; West et al. 2012). Several hypotheses were proposed for the shift in this pathogenic species. The main ones were the warming climate, increasing use of minimal or no tillage, the introduction of maize into the crop rotation species, and a general increase in spring wheat growing areas (Kelly et al. 2015; Mourelos et al. 2014; Parikka et al. 2012; Pereyra and Dill-Macky 2008; West et al. 2012).

As cereals become the permanent fixtures in crop rotations, the tracking of $F$. graminearum inocula sources also becomes very important for ensuring adequate control measures for the prevention of FHB outbreaks. F. graminearum overwinters on plant residues in soil and infects its hosts at the flowering stage (Leplat et al. 2013). In crop rotations, cereal crops are followed by noncereals, which are not primary hosts of $F$. graminearum, although some may act as an alternative host and harbor FHB agents (Mourelos et al. 2014; Pereyra and Dill-Macky 2008). Most of the FGSC studies focus on the primary hosts (wheat and barley), neglecting weeds, which are present in agriculture fields year to year regardless of the crops planted. The importance of weeds for the survival and spread of $F$. graminearum should not be ignored.

This study involved surveying crop fields over the course of 3 years. During this period, we collected $F$. graminearum samples from different sources: (i) its primary hosts-wheat and barley, (ii) other crop rotation species-rapeseed and sugar beet, (iii) numerous weed species growing among crops, (iv) dead plant residue at the end of growing seasons, (v) samples of soil, and (vi) a batch of barley seed before planting them. We used variable number tandem repeat (VNTR) markers to estimate the genetic diversity of $F$. graminearum and to analyze the genetic structure of the population. The objective of the present study was to evaluate chemotype distribution, clonal composition, and genetic variation differences in tested multiple isolation sources. 


\section{MATERIALS AND METHODS}

Study site description. This study was performed at six different crop rotation fields, where cereal crops (barley and wheat) were rotated with noncereal plants (pea, rapeseed, potato, and sugar beet) (Table 1). All fields were in close proximity to each other; at any given site, two fields were no more than $1 \mathrm{~km}$ apart. Thus, on a large geographical scale, all fungal isolates from these fields were expected to be representatives of one local population.

Isolate purification. Isolation of $F$. graminearum was performed from several different sources: its primary hosts (cereal crops), different weed species growing in fields among the crops, dead and decaying plant matter, samples of soil, and two batches of seed that were used to plant crops in 2017. When sampling crops, only ears with disease symptoms were collected for the isolation of fungi. In the case of weeds and decaying plants, asymptomatic plants were randomly collected, and isolation was performed from different plant parts (root, crown, stem, leaf, flower, or fruit). Plants of the same weed species were picked in different field sites, avoiding the collection of weeds of the same species growing in clusters.

Fungi from soil samples were isolated using the dilution plating method. A soil sample $(10 \mathrm{~g})$ was suspended in $100 \mathrm{ml}$ of sterile distilled water. The suspension was then diluted from $10^{-1}$ to $10^{-5}$. Dilutions ( $1 \mathrm{ml}$ of $10^{-5}$ to $10^{-3}$ ) were uniformly dispensed under the surface of malt agar in Petri dishes and incubated for 3 to 5 days at $24^{\circ} \mathrm{C}$ in the dark. Seed or plant segments of approximately $1 \mathrm{~cm}$ in length were surface sterilized in $1 \% \mathrm{NaOCl}$ for $3 \mathrm{~min}$, rinsed three times in sterile distilled water, dried out, plated on potato dextrose agar (PDA) (Sigma-Aldrich $\mathrm{GmbH}$, Taufkirchen, Germany) and incubated for 3 to 5 days at $24^{\circ} \mathrm{C}$ in the dark (Mathur and Kongsdal 2003). Growing Fusarium colonies were isolated on synthetic nutrient-poor agar, incubated for 3 to 5 days at $24^{\circ} \mathrm{C}$ in the dark until a macroconidial mass was formed, and preliminarily identified according to the manual of Leslie and Summerell (2006). $F$. graminearum spore suspensions in sterile water were spread onto $2 \%$ water agar and germinated. Single spores were picked and transferred onto PDA to obtain pure cultures for subsequent DNA extraction.

DNA extraction. DNA for PCR was extracted from 1- to 2week-old mycelium using a ZR Fungal/Bacterial DNA MiniPrep kit (Zymo Research, Irvine, CA, U.S.A.). The procedure was performed according to manufacturer's instructions. Samples were homogenized using a FastPrep 24 5G (MP Biomedicals, Santa Ana, CA, U.S.A.) homogenizer.

PCR amplification. Initially, isolates were identified as members of the FGSC by PCR using the Fg16F/Fg16R primer pair (Nicholson et al. 1998). Later, all of these isolates were subjected to translation elongation factor- $1 \alpha$ gene-based PCR method described by Yang et al. (2008), and confirmed as $F$. graminearum sensu stricto. Trichothecene genotypes were determined by PCR using the Tri303F/Tri303R, Tri315F/ Tri315R, and TriNivF/TriNivR primer pairs (Jennings et al. 2004). Genotyping was performed using VNTR PCR primers (Suga et al. 2004). All reactions were performed in mixtures containing $2.5 \mu \mathrm{l}$ of $10 \times$ PCR buffer (provided with the polymerase; Applied Biosystems, Waltham, MA, U.S.A.), $0.5 \mu l$ of dNTP Mix (10 mM each) (Thermo Fisher Scientific Baltics, Vilnius, Lithuania), $0.5 \mu \mathrm{l}$ of each $25 \mu \mathrm{M}$ primer, $1.25 \mathrm{U}$ of AmpliTaq Gold polymerase (Applied Biosystems), $1 \mu \mathrm{l}$ of extracted DNA template, and nuclease-free water up to a total volume of $25 \mu \mathrm{l}$. The thermocycling conditions consisted of initial denaturation and polymerase activation at $95^{\circ} \mathrm{C}$ for $10 \mathrm{~min}$; then, 38 cycles of $95^{\circ} \mathrm{C}$ for $40 \mathrm{~s}, 55$ to $62^{\circ} \mathrm{C}$ for $30 \mathrm{~s}$, and $72^{\circ} \mathrm{C}$ for $55 \mathrm{~s}$; followed by a final extension at $72^{\circ} \mathrm{C}$ for $10 \mathrm{~min}$. The annealing temperature was selected for each primer pair according to their original description.

Electrophoresis. Electrophoresis was carried out using a Cleaver MultiSub Mini apparatus (Cleaver Scientific Ltd., Rugby, Warwickshire, U.K.). The PCR products of species and trichothecene genotype identification were observed in $1 \%$ agarose gels using a GeneRuler 100-bp DNA Ladder (Thermo Fisher Scientific Baltics) as a marker. VNTR genotype PCR products were visualized in high-resolution 3\% MetaPhor (Lonza, Basel, Switzerland) agarose gels with GeneRuler Low-Range DNA Ladder and phiX174 DNA/BsuRI (HaeIII) markers (Thermo Fisher Scientific Baltics).

Data analysis. Data for population genetic analyses were generated using VNTR markers. These markers are composed of 10 loci of noncoding sequences that are dispersed throughout the entire genome. Genotypic richness, represented by the number of observed multilocus genotypes (MLG) and expected MLG (eMLG), as well as genotypic evenness (E.5) and genotypic diversity in the form of Simpson's index $(\lambda)$ and Nei's unbiased gene diversity (Hexp), respectively, were calculated from the raw data set in the poppr package of the $\mathrm{R}$ statistical language, v3.6.0 (Kamvar et al. 2015; R Core Team 2018). The same software was used to test VNTR marker discriminatory power by calculating the genotype accumulation curve using 1,000sample randomization (raw data set) and to test linkage disequilibrium with a standardized index of association (rbarD) using 999 permutations (both the raw and clone-corrected data sets). The standard poppr procedure of rbarD calculation was performed; thus, it involved the sample size correction using rarefaction. Clone correction was defined as the removal of redundant copies of each MLG within each population so that only a single copy remained and was also performed with poppr package of R. GenAlEx, v6.502 (Peakall and Smouse 2012) was used with the raw data set to conduct a principle coordinates analysis (PCoA) based on the pairwise genetic distances for haploid data (Huff et al. 1993) to calculate percentage of polymorphic loci (\%P), unbiased genetic diversity (uh), and alleles unique to certain populations. The population differentiation was evaluated using $\mathrm{F}_{\mathrm{ST}}$ between population pairs. Pairwise $\mathrm{F}_{\mathrm{ST}}$ estimates were based on the number of different alleles. The statistical significance of each pairwise $F_{S T}$ was tested by 10,000 permutations and at the 0.05 confidence level after Bonferroni correction. $\mathrm{F}_{\mathrm{ST}}$ analysis was carried out on both the raw and clone-corrected data sets. However, only results of the raw data are presented in publication because clone correction reduced the number of individuals in some groups (gr7, gr11, and gr13) to the extent where significance of statistical analysis becomes questionable. The hierarchical analysis of molecular variance (AMOVA) (Excoffier et al. 1992 ) with 10,000 permutations and pairwise $F_{\text {ST }}$ was estimated using Arlequin, v3.5.2.2 (Excoffier et al. 2005), using raw and clone-corrected data. The unconditional exact test was performed to compare trichothecene chemotype frequencies.

TABLE 1. Crop rotation in test fields (I to VI) ${ }^{\mathrm{a}}$

\begin{tabular}{|c|c|c|c|c|c|c|}
\hline Year & I (CT) & II (CT) & III (NT) & IV (CT) & $\mathrm{V}(\mathrm{CT})$ & VI (CT) \\
\hline 2015 & Pea & Pea & Pea & Spring rape & Sugar beet & Potato \\
\hline 2016 & Winter wheat & Winter wheat & Winter wheat & Spring wheat & Spring wheat & Spring barley \\
\hline 2017 & Spring barley & Winter rape & Winter rape & Spring barley & Spring barley & Winter wheat \\
\hline
\end{tabular}

${ }^{\mathrm{a}} \mathrm{CT}=$ conventional tillage and $\mathrm{NT}=$ no tillage. 


\section{RESULTS}

F. graminearum isolates. During this study, we obtained 804 isolates of $F$. graminearum. Crops were sampled over 2 years; 94 and 117 isolates were obtained in 2016 (gr1) and 2017 (gr2), respectively. More than 800 weed plants-representing more than 50 species-were collected during 3 years. From these, 58, 153, and 195 F. graminearum isolates were obtained in 2015 (gr3), 2016 (gr4), and 2017 (gr5), respectively. Decaying plant residue samples were collected over 4 years; $43,13,18$, and 0 isolates were found in 2014 (gr6), 2015 (gr7), 2016 (gr8), and 2017, respectively. Finally, 0, 21, and 3 isolates were found in soil samples from 2015, 2016 (gr9), and 2017, respectively. We chose to omit three isolates acquired from the soil in 2017 from further statistical analyses because the number was too low to represent a separate group. Additionally, we acquired 26 isolates from sugar beet (gr12) that were growing close to the test fields. These isolates were considered to be representatives of the same larger local population. Furthermore, 46 isolates were obtained from a batch of barley seed sown in test fields in 2017. Two types of seed were used: commercially acquired and those harvested from crops of the previous season grown in the same area. Of these 46 seedborne $F$. graminearum isolates, 19 were found in harvested seed (gr10) and 27 in bought seed (gr11). For comparison purposes, 20 isolates from crops were brought from the neighboring country, Latvia (gr13), and included in the analysis. In total, we distinguished 13 sources of isolates, 11 of which were expected to be from one local population (gr1 to gr10 and gr12) and two from different populations (gr11 and gr13).

Notably, 10 to 20 soil samples and, on average, 100 plant residue samples were taken from each of the six test fields every year. Furthermore, we collected $F$. graminearum from different parts of plants, and its isolation rate from parts that were higher on the plant vertical scale (leaves and upper part of the stem) was much greater than the isolation rate from ground-level parts; namely, roots, crown, and lower parts of the stem (data not shown).

VNTR alleles. Initially, we used all 10 primer pairs described by Suga et al. (2004). After genotyping the first 100 isolates, two primer pairs (HK630 and HK967 loci) were excluded from further analysis because they generated no polymorphism. The remaining eight markers were highly variable and produced between 4 and 19 alleles at each locus (mean $=8$ ) (Table 2). The highest number of alleles (19 alleles) was established at the HK957 locus, followed by the HK1073 locus (10 alleles). The highest uh values (0.848 and 0.802) were calculated for HK957 and HK1073, respectively, whereas the lowest $(0.288$ and 0.369$)$ were calculated for the HK913 and HK1043 loci, respectively. The \% P our chosen eight VNTR loci in the analyzed groups was $100 \%$, except for gr8 and gr11 $(\% \mathrm{P}=87.5 \%)$, for which HK1043 and HK913 loci, respectively, were monomorphic.

To determine the sufficient number of loci that could distinguish our sample pool size, we tested the discriminatory power of VNTR

TABLE 2. Number of alleles and gene diversity of eight variable number of tandem repeat (VNTR) markers used to analyze 804 Fusarium graminearum isolates

\begin{tabular}{lcc}
\hline VNTR locus & Number of alleles & uh $^{\mathrm{a}}$ \\
\hline HK957 & 19 & 0.848 \\
HK1073 & 10 & 0.802 \\
HK965 & 9 & 0.748 \\
HK917 & 7 & 0.677 \\
HK977 & 5 & 0.635 \\
HK1059 & 5 & 0.546 \\
HK913 & 4 & 0.369 \\
HK1043 & 5 & 0.288 \\
Mean & 8 & 0.614 \\
\hline
\end{tabular}

a Unbiased gene diversity. markers in poppr. The genotype accumulation curve in poppr is constructed up to $n-1$ loci sampled; thus, only seven loci are shown in the graph (Fig. 1). Seven markers covered slightly more than $90 \%$ of the unique genotypes observed. However, the curve did not plateau, which means that, in theory, the use of more markers could have resulted in detection of greater numbers of distinct genotypes. As mentioned, we discarded two genetic markers after they generated no polymorphism. They were tested on isolates of weeds in 2015 (gr3) and 2016 (gr4). Both groups showed overall high genetic diversity and low numbers of clones and, therefore, were good candidates to test marker polymorphism.

Trichothecene chemotypes. Of $804 \mathrm{~F}$. graminearum isolates, $589(73.3 \%)$ had the $15-$ ADON genotype and $215(26.7 \%)$ had the 3 -ADON genotype. No isolates of the NIV genotype were detected. Isolates from the commercially acquired barley seed had a very high (55.6\%) 3-ADON rate (15 of 27). Isolates from the locally harvested seed had a marginally high $3-A D O N$ rate $(42.1 \%)$ compared with all the other groups that had 3-ADON genotype rates ranging from 5.6 to $34.0 \%$ (Table 3 ).

Genotypic richness, diversity, and clonal composition. Of 804 sampled isolates, we found 619 unique MLGs. The eMLGs ranged from 8.85 to 10 in 11 of 13 sampled groups (Table 3 ). Comparatively, eMLG values in gr13 (Latvian crops) and gr11 (bought seed) were 7.23 and 6.43, respectively. Genotype distribution among samples within the tested groups was very close to equal abundance with E.5 values $>0.856$, except gr11, which stood out with a value of 0.470 . The same group displayed the lowest Hexp value (0.446); all other groups had values that ranged from 0.580 to 0.667 . The genotypic diversity calculated using $\lambda$ was lowest for gr11 (0.779), followed by gr13 (0.845) and gr7 (0.899), while the remaining groups showed $\lambda$ values $>0.941$.

The rbarD was used to assess the impact of clonal reproduction of F. graminearum on our data analysis. Based on the raw data, gr11 and gr13 stood out with relatively higher rbarD values of 0.3525 $(P=0.001)$ and $0.3269(P=0.001)$, respectively (Table 3$)$. These two groups were the only ones that did not belong to the study population: isolates from commercially acquired seed and crops from another country. All remaining groups (gr1 to gr10 and gr12) represented one local population. Overall, groups 1, 2, 4, 5, 6, 7, 11, and 13 seem to be in linkage disequilibrium. However, using clonecorrected data, we reject the null hypothesis that no linkage exists between markers only for groups 6 and 13, thus indicating high influence of asexual reproduction in these two groups. Remaining groups seem to be more in freely occurring recombination than

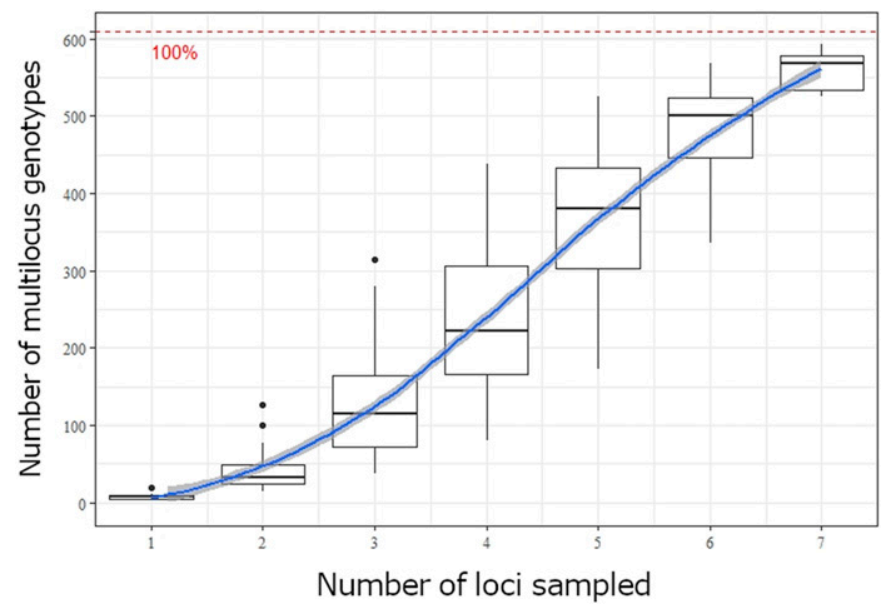

Fig. 1. Genotype accumulation curve for 804 isolates of Fusarium graminearum genotyped over eight loci. The horizontal axis represents the number of loci sampled (up to $n-1$ loci) and the vertical axis represents the number of unique multilocus genotypes observed. Data analyzed in poppr package for $\mathrm{R}$ with 1,000 sample randomization (Kamvar et al. 2015; R Core Team 2018). 
clonal reproduction, considering that $F$. graminearum can employ both procreation strategies and, thus, some amount of clones is always present in a population.

Population genetic structure. The overall $\mathrm{F}_{\mathrm{ST}}$ showed that population differentiation was low $(0.029)$ but significant $(P<$ 0.001 ). When gr11 (bought seed) and gr13 (Latvian crops) were excluded, $\mathrm{F}_{\mathrm{ST}}$ declined to $0.019(P<0.001)$. The detected pairwise $\mathrm{F}_{\mathrm{ST}}$ values clearly indicated the genetic divergence of gr11 and gr13 from all the other groups (Table 4). The harvested seed (gr10) and sugar beet (gr12) groups did not show any differentiation from the other groups of our local population (gr1 to gr9). In general, genetic divergence was established between gr1 to gr5 (growing plants) and gr6 to gr9 (soil biome).

The hierarchical AMOVA resulted in the highest among-group variation $(8.42 \%)$ when gr11 and gr13 were separated from all the remaining groups (Table 5). The analysis rejected temporal genetic

TABLE 3. Different sampling groups, acquired Fusarium graminearum isolates, their trichothecene genotypes, estimates of intrapopulation genetic diversity, and index of association

\begin{tabular}{|c|c|c|c|c|c|c|c|c|c|}
\hline \multirow[b]{2}{*}{ Sampling group } & \multirow[b]{2}{*}{$N^{\mathrm{a}}$} & \multirow[b]{2}{*}{$15 \mathrm{~A} / 3 \mathrm{~A}(\%)^{\mathrm{b}}$} & \multirow[b]{2}{*}{$\mathrm{MLG}^{\mathrm{c}}$} & \multirow[b]{2}{*}{$\mathrm{eMLG}^{\mathrm{d}}$} & \multirow[b]{2}{*}{ E. $5^{\mathrm{e}}$} & \multirow[b]{2}{*}{$\lambda^{\mathrm{f}}$} & \multirow[b]{2}{*}{$\operatorname{Hexp}^{\mathrm{g}}$} & \multirow{2}{*}{$\begin{array}{l}\mathrm{rbarD} / P^{\mathrm{h}} \\
\text { Raw data }\end{array}$} & \multirow[b]{2}{*}{ Clone-corrected } \\
\hline & & & & & & & & & \\
\hline gr1 (crops 2016) & 94 & $66.0 / 34.0$ & 82 & 9.84 & 0.908 & 0.986 & 0.615 & $0.0120 / 0.040$ & $0.0035 / 0.280$ \\
\hline gr2 (crops 2017) & 117 & $70.1 / 29.9$ & 99 & 9.84 & 0.891 & 0.988 & 0.642 & $0.0180 / 0.004$ & $0.0068 / 0.139$ \\
\hline gr3 (weeds 2015) & 58 & $79.3 / 20.7$ & 54 & 9.89 & 0.966 & 0.980 & 0.614 & $0.0031 / 0.375$ & $-0.0030 / 0.582$ \\
\hline gr4 (weeds 2016) & 153 & $75.2 / 24.8$ & 138 & 9.91 & 0.889 & 0.991 & 0.635 & $0.0091 / 0.044$ & $0.0010 / 0.403$ \\
\hline gr5 (weeds 2017) & 195 & $76.4 / 23.6$ & 150 & 9.84 & 0.857 & 0.991 & 0.617 & $0.0177 / 0.001$ & $0.0080 / 0.051$ \\
\hline gr6 (residue 2014) & 43 & $67.4 / 32.6$ & 33 & 9.42 & 0.905 & 0.964 & 0.654 & $0.0805 / 0.001$ & $0.0359 / 0.004$ \\
\hline gr7 (residue 2015) & 13 & $76.9 / 23.1$ & 11 & 8.85 & 0.941 & 0.899 & 0.663 & $0.0572 / 0.014$ & $0.0315 / 0.104$ \\
\hline gr8 (residue 2016) & 18 & $94.4 / 5.6$ & 18 & 10 & 1.000 & 0.946 & 0.580 & $0.0096 / 0.385$ & $0.0096 / 0.388$ \\
\hline gr9 (soil 2016) & 21 & $71.4 / 28.6$ & 21 & 10 & 1.000 & 0.952 & 0.589 & $0.0129 / 0.199$ & $0.0129 / 0.204$ \\
\hline gr10 (harvest seed) & 19 & $57.9 / 42.1$ & 18 & 9.74 & 0.972 & 0.942 & 0.667 & $-0.0009 / 0.530$ & $-0.0110 / 0.718$ \\
\hline gr11 (bought seed) & 27 & $44.4 / 55.6$ & 15 & 6.43 & 0.470 & 0.779 & 0.446 & $0.3525 / 0.001$ & $0.0392 / 0.108$ \\
\hline gr12 (beet 2017) & 26 & $88.5 / 11.5$ & 26 & 10 & 1.000 & 0.962 & 0.653 & $0.0098 / 0.221$ & $0.0098 / 0.233$ \\
\hline gr13 (crops, Latvia) & 20 & $90.0 / 10.0$ & 13 & 7.23 & 0.866 & 0.845 & 0.608 & $0.3269 / 0.001$ & $0.1280 / 0.015$ \\
\hline Overall & 804 & $73.3 / 26.7$ & 619 & 9.93 & 0.676 & 0.997 & 0.633 & $0.0107 / 0.001$ & $0.0064 / 0.005$ \\
\hline
\end{tabular}

a Number of isolates.

b $15 \mathrm{~A}=15$-acetyldeoxynivalenol and $3 \mathrm{~A}=3$-acetyldeoxynivalenol.

c Number of multilocus genotypes observed.

d Number of expected MLGs at the smallest sample size based on rarefaction.

e Evenness.

f Simpson's index.

g Nei's unbiased gene diversity.

${ }^{\mathrm{h}}$ rbarD $=$ standardized index of association and its statistical significance ( $P$ value).

TABLE 4. Genetic differentiation for Fusarium graminearum population pairs ${ }^{\mathrm{a}}$

\begin{tabular}{|c|c|c|c|c|c|c|c|c|c|c|c|c|c|}
\hline Sampling group & gr1 & gr2 & gr3 & $\mathrm{gr} 4$ & gr5 & gr6 & gr7 & gr8 & gr9 & gr10 & gr11 & gr12 & $\operatorname{gr} 13$ \\
\hline gr1 (crops 2016) & $\ldots$ & 0.150 & 0.010 & 0.593 & 0.056 & 0.000 & 0.003 & 0.037 & 0.001 & 0.147 & 0.000 & 0.499 & 0.005 \\
\hline gr2 (crops 2017) & 0.078 & $\ldots$ & 0.002 & 0.004 & 0.235 & 0.000 & 0.006 & 0.104 & 0.002 & 0.385 & 0.000 & 0.314 & 0.000 \\
\hline gr3 (weeds 2015) & 0.012 & 0.023 & $\ldots$ & 0.522 & 0.016 & 0.000 & 0.000 & 0.034 & 0.000 & 0.142 & 0.000 & 0.431 & 0.001 \\
\hline gr4 (weeds 2016) & 0.003 & 0.012 & 0.005 & $\ldots$ & 0.010 & 0.000 & 0.002 & 0.039 & 0.000 & 0.376 & 0.000 & 0.750 & 0.002 \\
\hline gr5 (weeds 2017) & 0.008 & 0.005 & 0.014 & 0.008 & $\ldots$ & 0.000 & 0.006 & 0.148 & 0.001 & 0.736 & 0.000 & 0.760 & 0.000 \\
\hline gr6 (residue 2014) & 0.038 & 0.037 & 0.055 & 0.033 & 0.030 & $\ldots$ & 0.229 & 0.147 & 0.026 & 0.169 & 0.000 & 0.029 & 0.001 \\
\hline gr7 (residue 2015) & 0.069 & 0.062 & 0.080 & 0.063 & 0.059 & 0.036 & $\ldots$ & 0.154 & 0.071 & 0.259 & 0.000 & 0.048 & 0.004 \\
\hline gr8 (residue 2016) & 0.065 & 0.051 & 0.067 & 0.059 & 0.045 & 0.056 & 0.083 & $\ldots$ & 0.978 & 0.451 & 0.005 & 0.280 & 0.028 \\
\hline gr9 (soil 2016) & 0.062 & 0.050 & 0.062 & 0.054 & 0.045 & 0.047 & 0.067 & -0.001 & $\ldots$ & 0.297 & 0.000 & 0.003 & 0.002 \\
\hline gr10 (harvest seed) & 0.025 & 0.016 & 0.028 & 0.017 & 0.008 & 0.030 & 0.044 & 0.042 & 0.032 & $\ldots$ & 0.001 & 0.787 & 0.004 \\
\hline gr11 (bought seed) & 0.117 & 0.097 & 0.111 & 0.105 & 0.088 & 0.159 & 0.201 & 0.164 & 0.162 & 0.117 & $\ldots$ & 0.000 & 0.000 \\
\hline gr12 (beet 2017) & 0.012 & 0.014 & 0.015 & 0.007 & 0.006 & 0.039 & 0.060 & 0.048 & 0.066 & 0.013 & 0.123 & $\ldots$ & 0.011 \\
\hline gr13 (Latvia) & 0.080 & 0.096 & 0.089 & 0.078 & 0.102 & 0.113 & 0.150 & 0.133 & 0.132 & 0.110 & 0.246 & 0.091 & $\ldots$ \\
\hline
\end{tabular}

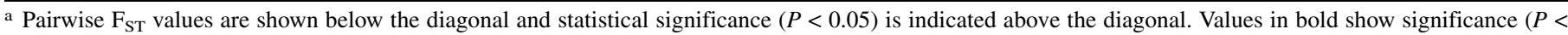
$0.004)$ after Bonferroni correction.

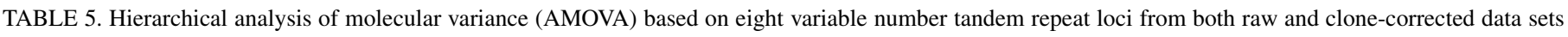

\begin{tabular}{|c|c|c|c|c|}
\hline \multirow[b]{2}{*}{ Grouping } & \multirow[b]{2}{*}{ Sampling group } & \multicolumn{3}{|c|}{ Percentage of variation raw/clone corrected data ${ }^{a}$} \\
\hline & & Groups & Among pop. & Within pop. \\
\hline By location & $\begin{array}{l}\text { [gr11 (bought seed)] [gr13 (Latvia)] [all } \\
\text { remaining groups] }\end{array}$ & $8.42 / 4.68$ & $1.75 / 1.68$ & $89.83 / 93.74$ \\
\hline Local, by source & $\begin{array}{l}\text { [gr1, gr2 (crops)] [gr3-gr5 (weeds)] [gr6-gr8 } \\
\text { (residue)] [gr9 (soil)] [gr10 (harvest seed)] }\end{array}$ & $1.01 / 0.71$ & $1.20 / 1.12$ & $97.79 / 98.16$ \\
\hline Local, by time & $\begin{array}{l}\text { [gr6 (2014)] [gr3, gr7 (2015)] [gr1, gr4, gr8 } \\
\quad(2016)][\text { gr2, gr5 (2017)] }\end{array}$ & 0.40 n.s./0.27 n.s. & $1.35 / 1.13$ & $98.25 / 98.60$ \\
\hline Only growing plants & $\begin{array}{l}\text { [gr1, gr2 (crops)] [gr3-gr5 (weeds)] [gr12 } \\
\text { (beet)] }\end{array}$ & -0.14 n.s./-0.09 n.s. & $1.07 / 0.89$ & $99.06 / 99.20$ \\
\hline Growing plants against soilborne & [gr1-gr5] [gr6-gr9] & $2.45 / 2.32$ & $1.45 / 1.07$ & $96.30 / 96.81$ \\
\hline
\end{tabular}

${ }^{\text {a }}$ Groups = among groups, Among pop. = among populations within groups, Within pop. $=$ within populations, and n.s. $=$ not significant $(P>0.05)$. 
differentiation as well as differentiation among the groups from growing plants (crops, weeds, and beet). By contrast, we noted significant $(P<0.05) 2.45 \%$ (raw data set) and $2.32 \%$ (after clonecorrection) variations when comparing isolates from growing plants against isolates from the soil biome.

The PCoA demonstrated genetic remoteness of two groups (namely, commercially acquired seed and isolates found on Latvian crops) and close clustering of all groups from the test area (data not shown). The removal of genetically distant groups allowed us to observe subpopulation relationships (Fig. 2). Isolates from plants that grew during a season were more closely related compared with isolates from dead plant residue and soil, which were both collected at the end of the growing season. Furthermore, we observed close clustering by year. Isolates from weeds and crops were the most closely related year by year.

\section{DISCUSSION}

To our knowledge, this study provides the first attempt to investigate the $F$. graminearum population structure by examining multiple distinct (but spatially related) sources of isolates: primary and secondary crop hosts, weeds, plant residue, soil, and seed. Samples were gathered over 3 years to also assess the temporal effect. The evaluated site comprised different fields to address distinct crop hosts and tillage because this study was part of a larger investigation. This design does not affect genetic structure of the population. All test fields were expected to represent one local F. graminearum population because of their close proximity. Two outside groups (gr11 and gr13) were added to have separate populations serve as significantly different comparison points.

$F$. graminearum isolation rates from crops were nearly $100 \%$ because only crop ears exhibiting disease symptoms were collected. Weeds, plant residue, soil, and seed were tested randomly. From more than 800 tested weed plants, we identified 406 isolates, for an approximately $50 \%$ isolation rate. By contrast, from over 300 soil samples, we found only 24 isolates, a $<10 \%$ isolation rate. Furthermore, 74 isolates were acquired from 1,850 tested plant residue samples, a 4\% isolation rate. $F$. graminearum is reportedly not a very competitive organism when it comes to survival in decaying crop residues and soil (Leplat et al. 2013). Despite this, there is a long-standing belief that plant residues are the primary source of infection inoculum. The low amount of $F$. graminearum isolated from soil and plant residue samples compared with the high amount in weeds, as well as the higher isolation rate from upper versus lower plant parts, raises the question of how significant is the role of soil as the primary infection source in spring compared with plants around the agricultural fields.

Overall, $73.3 \%$ of the isolates had the 15-ADON genotype and $26.7 \%$ had the 3 -ADON genotype. The chemotype ratio remained nearly the same when we examined only the local population

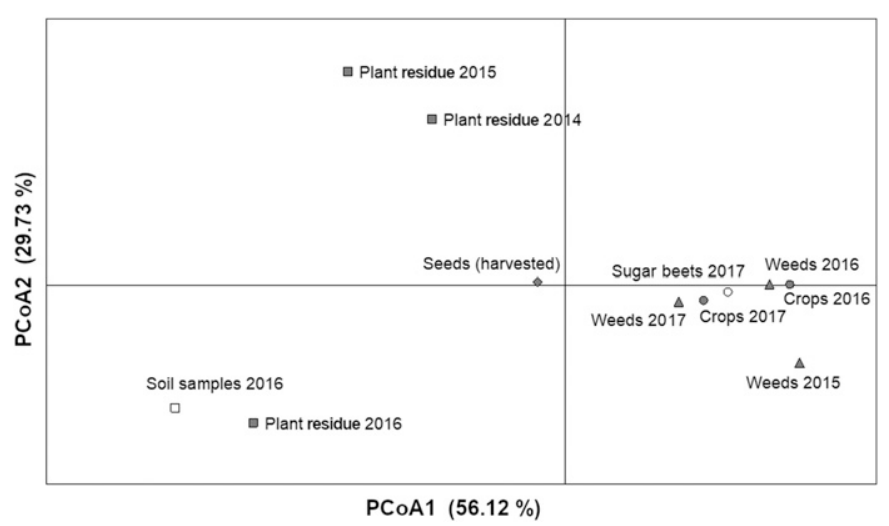

Fig. 2. Principal coordinate analysis (PCoA) of genetic distances between Fusarium graminearum groups from different sources of one local population. The plot of the first two axes explains $85.85 \%$ of genetic variation. (excluding gr11 and gr13); namely, 73.8 and 26.2\%, respectively. The highest ratio of 3-ADON was in two groups of isolates that were obtained from seed: 55.6 and $42.1 \%$ for gr 11 (bought seed) and gr10 (harvested seed), respectively (Table 3). Based on the unique genotypes observed from the total number of samples, the boughtseed group had the highest ratio of clones (45\%); this factor affected the observed chemotype percentage. In contrast, based on genetic richness and E.5, isolates from harvested seed displayed high genetic variability as well as a low number of clonal individuals. A previous study reported that $15-\mathrm{ADON}$ is clearly dominating in $F$. graminearum isolates in Lithuanian crops (Suproniene et al. 2016). Concomitantly, the 3-ADON genotype occurrence matches up or even exceeds 15-ADON in cases when homogenized seed are tested (Suproniene et al. 2016). Our results also hint at that trend; however, an unconditional exact test shows that $42.1 \%$ of the 3ADON content in harvested seed was not significantly higher $(P>$ 0.05 ) than the majority of our other tested groups (from 20.7 to $34 \%$ ). Notably, 3-ADON is reportedly more frequent only in Northern Europe, while 15-ADON is dominant over the 3-ADON and NIV genotypes in the rest of European $F$. graminearum populations (Aamot et al. 2015; Aoki et al. 2012; Boutigny et al. 2014; Bryła et al. 2016; Pasquali and Migheli 2014; Talas et al. 2011).

The bought-seed population was characterized by the lowest genetic variation based on most parameters (eMLG, $\lambda$, E.5, and Hexp). The low genetic diversity can be explained by a high number of infected seed that came from a single barley ear and were accidentally packed together in one bag. Such seed would be infected with clones or offspring of a single individual fungus. More interestingly, isolates from commercially acquired seed not only had a noticeably different trichothecene genotype composition but also the raw data revealed that this group had a high number of certain alleles that were extremely rare or not present in any other tested group (data not shown). For a long time, infected seed were considered to be a viable source of $F$. graminearum infection (Duthie and Hall 1987; Kabeere et al. 1997). More recent studies showed that infection may hinder seed germination and that some barriers exist which prevent fungus from infecting wheat heads during its systemic growth inside the plant (Hassani et al. 2019; Moretti et al. 2014). Our results are in agreement with the later findings. These seed were used to plant barley for the 2017 season in one of our test fields. However, the genetic properties of $F$. graminearum isolated later from this barley field did not correlate with the fungal isolates from the seed used.

The group of Latvian isolates was also characterized as highly clonal, based on rbarD values, whereas the tested local population displayed high genetic variation and lower rbarD values. Considering the number of unique genotypes observed in the collected samples, our data set had $23 \%$ of the possible clones. Comparing isolates from 2016 (gr1) and 2017 (gr2) crops, we found five recurrent MLGs. Because gr1 had 82 unique MLGs (Table 3), five of them carrying over to the next year accounted for $6 \%$. Similarly, in weed groups, gr3 had 54 MLGs and $3(5.6 \%)$ of them reoccurred next year in gr4, while gr4 had 138 MLGs and $8(5.8 \%)$ of them were found in gr5. Thus, our results indicated a tendency that approximately $6 \%$ of genotypes were carried from one year to the next. Other studies of plant-pathogenic fungi often report much higher amounts of suspected clones (>50\%) (Mercier et al. 2019; Sumabat et al. 2018; Walker et al. 2015). However, it is debatable whether it is valid to compare clonal composition of one population of pathogenic fungi to another, both within one species or between several different ones. Numerous reasons can affect the number of detectable clones in population: (i) sample collection strategy can be emphasized on avoidance of picking samples close to each other to a various degrees; (ii) plant rotations of our test fields comprised several different host species, whereas study of a single crop species could detect many more clones because a pathogen would be driven to specialize to a single host; and (iii) unique genotypes are 
predicted from genetic markers used, which (despite the laborious process of their creation) test only few tiny nucleotide sequences in the vast genome and the use of other markers could indicate slightly different amount of MLGs even in the same population. Overall, in our opinion, relatively low clonal composition of a population is a result of more pronounced sexual reproduction, which creates more diversity and more adoptability to the environment.

When comparing isolates from bought seed (gr11) and Latvian crops (gr13) with groups that represented the local test population, pairwise $F_{\text {ST }}$ values ranged from 0.078 to 0.201 (Table 4). Karugia et al. (2009) compared two Japanese populations with one from China and reported $\mathrm{F}_{\mathrm{ST}}$ values of 0.233 and 0.251 . A similar $\mathrm{F}_{\mathrm{ST}}$ value (0.28) was obtained between two separate Norwegian populations (Aamot et al. 2015). Relatively low (0.03 to 0.08) but mainly statistically significant $\mathrm{F}_{\mathrm{ST}}$ values were observed when comparing four groups from the soil biome (gr6 to gr9) against groups from growing plants (gr1 to gr5 and gr12). We found minor $\mathrm{F}_{\mathrm{ST}}$ values that ranged from 0.003 to 0.023 when comparing between weeds (gr3 to gr5) and crops or beet groups (gr1 to gr2 and gr12) (Table 4). AMOVA supplemented the $\mathrm{F}_{\mathrm{ST}}$ analysis results. The genetic differentiation was not observed when comparing isolates from crops, beet, and weeds, or when grouping the local population by collection year (Table 5). By contrast, a low but significant $2.45 \%$ of total variation was attributed to differences between isolates from crops and weeds versus soilborne isolates. These tendencies persisted in both the raw and clone-corrected datasets. A recent study also reported low differentiation between $F$. graminearum populations in primary hosts and weeds, and concluded that several species of wild grasses are local and regional inoculum sources for this pathogen (Fulcher et al. 2019). Our findings are consistent with the abovementioned results. Notably, in the present study, the $F$. graminearum isolation rate from plant residue and soil was very low, and the size of soil biome samples only ranged from 13 to 43 . It is possible that differentiation between plant and soil groups could be more highly expressed with larger sample sizes, because this increase could generate more distinct MLGs in a soil group. Thus, future studies of possible genetic differentiation between $F$. graminearum isolates from growing plants and the soil biome are of interest.

PcoA supplemented $\mathrm{F}_{\mathrm{ST}}$ analysis and showed isolates from plants clustering together and separately from isolates from the soil biome. Furthermore, it illustrated subtle differences in tested local populations. Isolates from the soil and plant residue from 2016 were grouped together. Because both soil and plant residue samples were collected at the end of the growing season, it is possible that spores from plant residue had spread over the soil. However, the clustering of plant residue isolates from 2014 and 2015 together and distantly from the 2016 group is more difficult to explain. In theory, tillage can hide plant residue by burying it deeper into the ground, thus effectively removing the samples and providing a niche for fungal spores of other (not soilborne) origin to settle in the beginning of the next season. The population grouping observed also might be affected by the lack of isolates from the soil from 2014 and 2015, as well as relatively low number of total samples in each of the soil biome groups. Isolates from sugar beet were closely related to crop and weed isolates of the same year.

There was no temporal- or chemotype-related population structure in our study. The same conclusion was made by similar FGSC studies in North America, Europe, and China (Aamot et al. 2015; Burlakoti et al. 2011; Qiu et al. 2014). One important factor that should be considered in future research is that many previous FGSC population structure studies did not involve insight about data clonality that arises from mixed reproduction strategies of fungi.

To conclude, we examined $F$. graminearum population substructure by emphasizing, for the first time, multiple different origin sources; namely, distinct crop hosts, weeds, seed, soil, and decaying plant residue. We also tested three main trichothecene genotypes and gathered isolates over 3 years to address temporal effects. We believe that our results scratched the surface of a delicate and barely noticeable - but logically possible $-F$. graminearum subpopulation division between aerial and soilborne spread strategies. Currently, there are no population structure studies that focused on differentiation of these two stages (actively infecting and saprotrophic survival) of any fungal plant pathogen. However, it might be similar to the specialization to plant hosts of some other fungal pathogens. Various plants may force a fungus to employ different strategies via expression of particular genes. For several plant-pathogenic fungi, there is noticeable subpopulation differentiation among isolates acquired from different primary hosts while, at the same time, there are no temporal or spatial divisions (Sumabat et al. 2018; Walker et al. 2015). Furthermore, any given local population may comprise the mixture of both the generalist and specialized fungus micropopulations (Mercier et al. 2019). In the case of F. graminearum, minor-but statistically significant-differentiation of plantinfecting and soilborne stages should be considered in contrast to no differentiation between isolates from crops and weeds. In the present study, there was an absence of any barriers among subpopulations of different plant hosts, data that support the recent findings of Fulcher et al. (2019). The lack of differentiation between pathogen communities in primary and nonprimary plant hosts shows the importance of weeds and other segetal plants that grow inside or in close proximity to agricultural fields. Such plants act as alternative hosts for $F$. graminearum and provide an additional means of spreading and survival, as well as the pool for genetic recombination. $F$. graminearum isolation rates from soilborne sources were considerably lower and, thus, the subpopulation differences between growing plant and soilborne isolates deserve more attention with focused research that employs a specifically tailored sample collection strategy.

\section{ACKNOWLEDGMENTS}

We thank the reviewers and journal editors for providing crucially insightful and constructive feedback that helped to significantly improve our manuscript.

\section{LITERATURE CITED}

Aamot, H. U., Ward, T. J., Brodal, G., Vralstad, T., Larsen, G. B., Klemsdal, S. S., Elameen, A., Uhlig, S., and Hofgaard, I. S. 2015. Genetic and phenotypic diversity within the Fusarium graminearum species complex in Norway. Eur. J. Plant Pathol. 142:501-519.

Aoki, T., Ward, T. J., Kistler, H. C., and O’Donnell, K. 2012. Systematics, phylogeny and trichothecene mycotoxin potential of Fusarium head blight cereal pathogens. Mycotoxins 62:91-102.

Boutigny, A. L., Ward, T. J., Ballois, N., Iancu, G., and Ioos, R. 2014. Diversity of the Fusarium graminearum species complex on French cereals. Eur. J. Plant Pathol. 138:133-148.

Bryła, M., Waśkiewicz, A., Podolska, G., Szymczyk, K., Jędrzejczak, R., Damaziak, K., and Sułek, A. 2016. Occurrence of 26 mycotoxins in the grain of cereals cultivated in Poland. Toxins (Basel) 8:160-180.

Burlakoti, R. R., Neate, S. M., Adhikari, T. B., Gyawali, S., Salas, B., Steffenson, B. J., and Schwarz, P. B. 2011. Trichothecene profiling and population genetic analysis of Gibberella zeae from barley in North Dakota and Minnesota. Phytopathology 101:687-695.

Duthie, J. A., and Hall, R. 1987. Transmission of Fusarium graminearum from seed to stems of winter wheat. Plant Pathol. 36:33-37.

Excoffier, L., Laval, G., and Schneider, S. 2005. Arlequin (version 3.0): An integrated software package for population genetics data analysis. Evol. Bioinf. 1:47-50.

Excoffier, L., Smouse, P. E., and Quattro, J. M. 1992. Analysis of molecular variance inferred from metric distances among DNA haplotypes: Application to human mitochondrial DNA restriction data. Genetics 131:479-491.

Fulcher, M. R., Winans, J. B., Quan, M., Oladipo, E. D., and Bergstrom, G. C. 2019. Population genetics of Fusarium graminearum at the interface of wheat and wild grass communities in New York. Phytopathology 109:2124-2131.

Hassani, F., Zare, L., and Khaledi, N. 2019. Evaluation of germination and vigor indices associated with Fusarium-infected seeds in pre-basic seeds wheat fields. J. Plant Prot. Res. 59:69-85. 
Huff, D. R., Peakall, R., and Smouse, P. E. 1993. RAPD variation within and among natural populations of outcrossing buffalograss Buchloe dactyloides (Nutt.) Engelm. Theor. Appl. Genet. 86:927-934.

Jennings, P., Coates, M. E., Turner, J. A., Chandler, E. A., and Nicholson, P. 2004. Determination of deoxynivalenol and nivalenol chemotypes of Fusarium culmorum isolates England and Wales by PCR assay. Plant Pathol. 53:182-190.

Kabeere, F., Hampton, J. G., and Hill, M. J. 1997. Transmission of Fusarium graminearum (Schwabe) from maize seeds to seedlings. Seed Sci. Technol. 25:245-252.

Kamvar, Z. N., Brooks, J. C., and Grünwald, J. 2015. Novel R tools for analysis of genome-wide population genetic data with emphasis on clonality. Front. Genet. 1:208.

Karugia, G. W., Suga, H., Gale, L. R., Nakajima, T., Ueda, A., and Hyakumachi, M. 2009. Population structure of Fusarium asiaticum from two Japanese regions and eastern China. J. Gen. Plant Pathol. 75: 110-118.

Kelly, A. C., Clear, R. M., O’Donnell, K., McCormick, S., Turkington, T. K., Tekauz, A., Gilbert, J., Kistler, H. C., Busman, M., and Ward, T. J. 2015. Diversity of Fusarium head blight populations and trichothecene toxin types reveals regional differences in pathogen composition and temporal dynamics. Fungal Genet. Biol. 82:22-31.

Leplat, J., Friberg, H., Abid, M., and Steinberg, C. 2013. Survival of Fusarium graminearum, the causal agent of Fusarium head blight. A review. Agron. Sustain. Dev. 33:97-111.

Leslie, J. F., and Summerell, B. A., eds. 2006. The Fusarium Laboratory Manual. Blackwell Publishing Ltd., Ames, IA, U.S.A.

Mathur, S. B., and Kongsdal, O. 2003. Common Laboratory Seed Health Testing Methods of Detecting Fungi.International Seed Testing Association (ISTA), Bassersdorf, Switzerland.

Mercier, A., Carpentier, F., Duplaix, C., Auger, A., Pradier, J. M., Viaud, M., Gladieux, P., and Walker, A. S. 2019. The polyphagous plant pathogenic fungus Botrytis cinerea encompasses host-specialized and generalist populations. Environ. Microbiol. 21:4808-4821.

Moretti, A., Panzarini, G., Somma, S., Campagna, C., Ravaglia, S., Logrieco, A. F., and Solfrizzo, M. 2014. Systemic growth of F. graminearum in wheat plants and related accumulation of deoxynivalenol. Toxins (Basel) 6: 1308-1324.

Mourelos, C. A., Malbran, I., Balatti, P. A., Ghiringhelli, P. D., and Lori, G. A. 2014. Gramineous and non-gramineous weed species as alternative hosts of Fusarium graminearum, causal agent of Fusarium head blight of wheat, in Argentina. Crop Prot. 65:100-104.

Nicholson, P., Simpson, D. R., Weston, G., Rezanoor, H. N., Lees, A. K., Parry, D. W., and Joyce, D. 1998. Detection and quantification of Fusarium culmorum and Fusarium graminearum in cereals using PCR assays. Physiol. Mol. Plant Pathol. 53:17-37.

Parikka, P., Hakala, K., and Tiilikkala, K. 2012. Expected shifts in Fusarium species' composition on cereal grain in Northern Europe due to climatic change. Food Addit. Contam. 29:1543-1555.

Pasquali, M., and Migheli, Q. 2014. Genetic approaches to chemotype determination in type B-trichothecene producing Fusaria. Int. J. Food Microbiol. 189:164-182.

Peakall, R., and Smouse, P. E. 2012. GenAlEx 6.5: Genetic analysis in Excel. Population genetic software for teaching and research-An update. Bioinformatics 28:2537-2539.
Pereyra, S. A., and Dill-Macky, R. 2008. Colonization of the residues of diverse plant species by Gibberella zeae and their contribution to Fusarium head blight inoculum. Plant Dis. 92:800-807.

Qiu, J., Xu, J., and Shi, J. 2014. Molecular characterization of the Fusarium graminearum species complex in Eastern China. Eur. J. Plant Pathol. 139:811-823.

R Core Team. 2018. R: A Language And Environment for Statistical Computing. R Foundation for Statistical Computing, Vienna, Austria. http:// www.r-project.org/index.html

Schothorst, R. C., and van Egmond, H. P. 2004. Report from SCOOP task 3.2.10 "collection of occurrence data of Fusarium toxins in food and assessment of dietary intake by the population of EU member states", Subtask: Trichothecenes. Toxicol. Lett. 153:133-143.

Somma, S., Petruzzella, A. L., Logrieco, A. F., Meca, G., Cacciola, O. S., and Moretti, A. 2014. Phylogenetic analyses of Fusarium graminearum strains from cereals in Italy, and characterization of their molecular and chemical chemotypes. Crop Pasture Sci. 65:52-60.

Suga, H., Gale, L. R., and Kistler, C. 2004. Development of VNTR markers for two Fusarium graminearum clade species. Mol. Ecol. Notes 4:468-470.

Sumabat, L. G., Kemerait, R. C., Kim, D. K., Mehta, Y. R., and Brewer, M. T. 2018. Clonality and geographic structure of host-specialized populations of Corynespora cassiicola causing emerging target spot epidemics in the southeastern United States. PLoS One 13:e0205849.

Suproniene, S., Sakalauskas, S., Stumbriene, K., Zvirdauskiene, R., and Svegzda, P. 2016. Variances in trichothecene chemotype distribution in Lithuanian wheat grain and within pure culture Fusarium graminearum isolated from the same grain samples. Eur. J. Plant Pathol. 144:371-381.

Talas, F., Parzies, H. K., and Miedaner, T. 2011. Diversity in genetic structure and chemotype composition of Fusarium graminearum sensu stricto populations causing wheat head blight in individual fields in Germany. Eur. J. Plant Pathol. 131:39-48.

Walker, A.-S., Gladieux, P., Decognet, V., Fermaud, M., Confais, J., Roudet, J., Bardin, M., Bout, A., Nicot, P. C., Poncet, C., and Fournier, E. 2015. Population structure and temporal maintenance of the multihost fungal pathogen Botrytis cinerea: Causes and implications for disease management. Environ. Microbiol. 17:1261-1274.

Wang, J. H., Ndoye, M., Zhang, J. B., Li, H. P., and Liao, Y. C. 2011. Population structure and genetic diversity of the Fusarium graminearum species complex. Toxins (Basel) 3:1020-1037.

West, J. S., Holdgate, S., Townsend, J. A., Edwards, S. G., Jennings, P., and Fitt, B. D. L. 2012. Impacts of changing climate and agronomic factors on fusarium ear blight of wheat in the UK. Fungal Ecol. 5:53-61.

Yang, L., Van der Lee, T. A. J., Yang, X., Yu, D., and Waalwijk, C. 2008. Fusarium populations on Chinese barley show a dramatic gradient in mycotoxin profiles. Phytopathology 98:719-727.

Yli-Mattila, T., Paavanen-Huhtala, S., Jestoi, M., Parikka, P., Hietaniemi, V., Gagkaeva, T., Sarlin, T., Haikara, A., Laaksonen, S., and Rizzo, A. 2008. Real-time PCR detection and quantification of Fusarium poae, F. graminearum, F. sporotrichioides and F. langsethiae in cereal grains in Finland and Russia. Arch. Phytopathol. Plant Prot. 41:243-260.

Yli-Mattila, T., Parikka, P., Lahtinen, T., Ramo, S., Kokkonen, M., Rizzo, A., Jestoi, M., and Hietaniemi, V. 2009. Fusarium DNA levels in Finnish cereal grains. Pages 107-138 in: Current Advances in Molecular Mycology. Y. Gherbawy, R. L. Mach, and M. K. Rai, eds. Nova Science Publishers, New York, NY, U.S.A. 